\title{
Detecting a secondary cosmic neutrino background from Majoron decays in neutrino capture experiments
}

\author{
Zackaria Chacko, ${ }^{1}$ Peizhi Du, ${ }^{1}$ and Michael Geller ${ }^{1,2}$ \\ ${ }^{1}$ Maryland Center for Fundamental Physics, Department of Physics, University of Maryland, \\ College Park, Maryland 20742-4111 USA \\ ${ }^{2}$ Department of Physics, Tel Aviv University, Tel Aviv 6997801, Israel
}

(Received 10 March 2019; published 30 July 2019)

\begin{abstract}
We consider theories in which the generation of neutrino masses is associated with the breaking of an approximate global lepton number symmetry. In such a scenario the spectrum of light states includes the Majoron, the pseudo-Nambu-Goldstone boson associated with the breaking of the global symmetry. For a broad range of parameters, the Majoron decays to neutrinos at late times, after the cosmic neutrinos have decoupled from the thermal bath, resulting in a secondary contribution to the cosmic neutrino background. We explore the possibility of directly detecting this secondary cosmic neutrino background in experiments based on neutrino capture on nuclei. For Majoron masses in the $\mathrm{eV}$ range or below, the neutrino flux from these decays can be comparable to that from the primary cosmic neutrino background, making it a promising target for direct detection experiments. The neutrinos from Majoron decay are redshifted by the cosmic expansion and exhibit a characteristic energy spectrum that depends on both the Majoron mass and its lifetime. For Majoron lifetimes of order the age of the Universe or larger, there is also a monochromatic contribution to the neutrino flux from Majoron decays in the Milky Way that can be comparable to the diffuse extragalactic flux. We find that for Majoron masses in the $\mathrm{eV}$ range, direct detection experiments based on neutrino capture on tritium, such as PTOLEMY, will be sensitive to this scenario with 100 gramyears of data. In the event of a signal, the galactic and extragalactic components can be distinguished on the basis of their distinct energy distributions, and also by using directional information obtained by polarizing the target nuclei.
\end{abstract}

DOI: $10.1103 /$ PhysRevD.100.015050

\section{INTRODUCTION}

While oscillation experiments have conclusively established that neutrinos have small but nonvanishing masses, the dynamics that generates these masses remains a mystery. In particular, it is not known whether neutrinos are Dirac or Majorana particles, whether their spectrum is hierarchical, inverse hierarchical or quasidegenerate, or why their masses are so small. Several ideas have been put forward to explain the origin of neutrino masses. These include the well-known seesaw mechanism [1-5] and the Majoron model [6-8].

Extensions of the standard model (SM) based on the Majoron framework incorporate a lepton number as a global symmetry. Neutrinos acquire masses once the global lepton number symmetry is spontaneously broken. The Majoron is the pseudo-Nambu-Goldstone boson associated

Published by the American Physical Society under the terms of the Creative Commons Attribution 4.0 International license. Further distribution of this work must maintain attribution to the author(s) and the published article's title, journal citation, and DOI. Funded by SCOAP ${ }^{3}$. with the breaking of the global symmetry. The form of its couplings to neutrinos is dictated by the nonlinearly realized lepton number symmetry. In the case when neutrinos are Dirac, the couplings of the Majoron field $J$ take the schematic form,

$$
i \frac{m_{\nu}}{f} J \nu n^{c}+\text { H.c. }
$$

where $\nu$ denotes the SM left-handed neutrino and $n^{c}$ its Dirac partner. Moreover, $m_{\nu}$ represents the neutrino mass, while $f$ denotes the Majoron decay constant, which corresponds to the scale at which the global lepton number symmetry is broken. In the case when the neutrino masses are Majorona, the corresponding coupling takes the form

$$
i \frac{m_{\nu}}{f} \frac{J}{2} \nu \nu+\text { H.c. }
$$

Equations (1) and (2) represent the leading order interactions of the Majoron with neutrinos in an expansion in $1 / f$. These couplings are diagonal in the basis in which the neutrino masses are diagonal. Hence the Majoron couples 
to each neutrino mass eigenstate with a strength proportional to the corresponding neutrino mass.

In general, the global symmetry need not be exact but only approximate, in which case the Majoron will acquire a mass. It then follows from the couplings of the Majoron to neutrinos, Eqs. (1) and (2), that any population of Majorons present in the early Universe will eventually decay to neutrinos, unless such decays are forbidden by phase space considerations. If the Majoron lifetime is greater than the age of the Universe, Majorons could constitute some or all of the observed dark matter of the Universe [9-13]. The required population of Majorons could have survived till the present day as thermal relics, or they could have been produced nonthermally through, for example, the misalignment mechanism [14-16]. If, however, the Majoron lifetime is less than or comparable to the age of the Universe, decays of the Majoron will result in a new contribution to the population of cosmic neutrinos. Provided the Majoron decays occur at late times, after the cosmic neutrinos have decoupled from the thermal bath, and at temperatures below the mass of the Majoron, this new population of neutrinos will be thermally decoupled and distinct from the cosmic neutrino background $(\mathrm{C} \nu \mathrm{B})$. Several authors have explored the possibility of detecting the neutrinos from Majoron decays in neutrino detectors such as Borexino, Super-Kamiokande and IceCube [17-19], as well as in dark matter detectors (see, e.g., [20]). In this paper we consider the decays of Majorons with masses of order an $\mathrm{eV}$ into neutrinos. We determine the current bounds on this scenario, and explore the possibility of directly detecting the resulting secondary $\mathrm{C} \nu \mathrm{B}$ in neutrino capture experiments.

Cosmological observations can be used to place limits on the flux of neutrinos from Majoron decay. These limits depend on the Majoron mass and lifetime. Decays that occur prior to recombination result in a contribution to the energy density in radiation that is present during the epoch of acoustic oscillations. Precision measurements of the cosmic microwave background (CMB) place severe limits on any such contribution, with the result that the number density of neutrinos from Majoron decays is always expected to be smaller than the $\mathrm{C} \nu \mathrm{B}$. In the case of Majorons that decay after recombination, the limits are much weaker. Current cosmological observations allow roughly $5 \%$ of the total energy density in dark matter to have decayed to dark radiation before today [21-23]. ${ }^{1}$ Then, if neutrinos are Majorana, the number density of neutrinos $\left(n_{\nu}^{J}\right)$ and antineutrinos $\left(n_{\bar{\nu}}^{J}\right)$ from Majoron decay is given by

\footnotetext{
${ }^{1}$ The bound on the fraction of dark matter that has decayed depends on the lifetime. The current bound indicates $\tau / \mathcal{F} \gtrsim$ 160 Gyr for $\tau \gtrsim t_{0}$ [21], where $\tau$ is the lifetime of the decaying dark matter while $\mathcal{F}$ denotes the fractional of energy density in decaying dark matter to that of all dark matter. Since we focus on the case in which the lifetime is of the order of the age of the Universe, we take the bound to be $\sim 5 \%$.
}

$$
n_{\nu}^{J}=n_{\bar{\nu}}^{J}=\mathcal{F} \Omega_{\mathrm{dm}} \frac{\rho_{c}}{m_{J}}\left(1-e^{-t_{0} / \tau_{J}}\right)
$$

In this expression $\tau_{J}$ represents the lifetime of the Majoron, and $m_{J}$ its mass. $\mathcal{F}$ denotes the fractional contribution of Majorons to the total energy density in dark matter at early times, while $\Omega_{\mathrm{dm}}$ is the fractional contribution of dark matter to the total energy density $\rho_{c}$. If the Majoron lifetime is much less than $t_{0}$, the age of the Universe, we have

$$
n_{\nu 0}^{J}=n_{\bar{\nu} 0}^{J}=\mathcal{F} \Omega_{\mathrm{dm}} \frac{\rho_{c}}{m_{J}}=\frac{63}{\mathrm{~cm}^{3}} \frac{\mathcal{F}}{5 \%} \frac{\mathrm{eV}}{m_{J}} .
$$

If instead neutrinos are Dirac, the number of active neutrinos and antineutrinos is half of this. For comparison, note that the total number density of the three flavors of neutrinos in the $\mathrm{C} \nu \mathrm{B}$ is $168 / \mathrm{cm}^{3}$, with an equal number of antineutrinos. We see from this that the number density of neutrinos arising from Majoron decays can be very large. In particular, for Majoron masses below an $\mathrm{eV}$, the flux from their decays can be comparable to or even exceed the flux from the cosmic background neutrinos. Therefore, the neutrinos from Majoron decays constitute a promising target for the direct detection experiments designed to detect the $\mathrm{C} \nu \mathrm{B}$.

Since there are only two particles in the final state, the neutrinos produced in Majoron decays are monochromatic. However, as a consequence of the cosmic expansion, these particles get redshifted, resulting in a characteristic energy distribution that depends not just on the Majoron mass, but also its width. If the Majoron lifetime is of the order of the age of the Universe, we also expect a flux of neutrinos from the decay of Majorons in the Milky Way galaxy. Since the time taken to traverse the galaxy is small compared to the age of the Universe, these neutrinos are monochromatic. The size of the galactic signal depends on the overdensity of Majorons in the Milky Way. If the Majorons are warm, as would be the case if they are thermal relics that were once in equilibrium with the SM, the overdensity is expected to be small. The galactic signal is then negligible. On the other hand, if the population of Majorons is very cold, as would be the case if they are produced through a misalignment mechanism, the overdensity is very large. In this case the distribution of Majorons in the galaxy is expected to follow the cold dark matter profile, resulting in a flux of galactic neutrinos that can be comparable to the diffuse extragalactic flux.

Experiments to detect the $\mathrm{C} \nu \mathrm{B}$ based on neutrino capture on tritium were first proposed by Weinberg more than 50 years ago [24]. Tritium undergoes beta decay with a half-life of 12.32 years,

$$
{ }^{3} \mathrm{H} \rightarrow{ }^{3} \mathrm{He}+e^{-}+\bar{\nu}_{e} .
$$

This process releases $18.6 \mathrm{keV}$ of energy, which is distributed between the final state electron and neutrino. However, this decay can be also induced by neutrino capture, 


$$
{ }^{3} \mathrm{H}+\nu_{e} \rightarrow{ }^{3} \mathrm{He}+e^{-} .
$$

The final state electron now carries away the energy of the incident neutrino, in addition to the energy liberated in the decay. Therefore, given sufficient energy resolution, searches for electrons with energies above the beta decay end point can be used for direct detection of cosmic neutrinos.

Recently, this idea has taken concrete shape in the form of the PTOLEMY experiment $[25,26]$, which aims to discover the $\mathrm{C} \nu \mathrm{B}$. PTOLEMY proposes to use $100 \mathrm{~g}$ of tritium coated on graphene. The energy resolution of PTOLEMY $\Delta$ is at the level of $0.15 \mathrm{eV}$ [25], which means that the experiment is not sensitive to neutrinos with energies below this threshold. Since the kinetic energies of cosmic neutrinos are typically negligible, in order to detect them, the neutrinos must be quasidegenerate, each with mass above $0.1 \mathrm{eV}$. A target of $100 \mathrm{~g}$ of tritium could detect about $4 \mathrm{C} \nu \mathrm{B}$ events per year if neutrinos are Dirac, rising to 8 events per year if neutrinos are Majorana [27]. ${ }^{2}$ However, the combined CMB and baryon acoustic oscillation (BAO) data indicate that the sum of neutrino masses $\sum m_{\nu}<0.12 \mathrm{eV}$ [29], which makes PTOLEMY unlikely to observe $\mathrm{C} \nu \mathrm{B}$ neutrinos.

In the case of Majorons with masses below an $\mathrm{eV}$, the flux of neutrinos from Majoron decay can be comparable to or larger than the flux from the $\mathrm{C} \nu \mathrm{B} .{ }^{3}$ We find that for Majoron masses in the $\mathrm{eV}$ range, direct detection experiments based on neutrino capture on tritium (e.g., PTOLEMY) will be sensitive to this scenario with 100 gramyears of data even if the SM neutrinos are lighter than $0.1 \mathrm{eV}$. Apart from an effect on the total event rate, we expect a distinctive energy spectrum of events inherited from the characteristic energy distributions of the neutrinos from extragalactic and galactic decays. These signals can be distinguished from the $\mathrm{C} \nu \mathrm{B}$, because the $\mathrm{C} \nu \mathrm{B}$ neutrinos are all nonrelativistic today.

Interestingly, by employing polarized tritium nuclei as the target [33], we can also obtain directional information about the neutrino flux. Since galactic Majorons are expected to follow the dark matter distribution in the Milky Way, the monochromatic component of the neutrino flux encodes information about the profile of dark matter in the galaxy. Therefore, by observing the modulation of the event rate with the orientation of the tritium spin, we can shed light on the galactic dark matter distribution.

This paper is organized as follows. We introduce the Majoron model in Sec. II and discuss the current bounds on this class of theories. In Sec. III we study the neutrino flux from Majoron decay, including both extragalactic and

\footnotetext{
${ }^{2}$ If the energy resolution is improved, it may be possible to obtain a signal even if neutrinos are not quasidegenerate. The event rate can then be sensitive to whether the lightest neutrino is massless [28].

${ }^{3}$ For models of new physics that alter the number density of nonrelativistic cosmic neutrinos, see e.g., [30-32].
}

galactic sources. In Sec. IV, we estimate the signal rates at PTOLEMY based on two benchmark points. We also study the dependence of the signal on the orientation of the spin of the tritium nuclei in the case of a polarized target. We conclude in Sec. V.

\section{THE MAJORON MODEL}

As the Goldstone boson associated with the breaking of lepton number, the form of the Majoron couplings in the low energy theory is dictated by the nonlinearly realized symmetry. If neutrinos are Dirac, below the symmetry breaking scale $f$, the coupling of the Majoron to leptons takes the schematic form

$$
i \lambda \frac{J}{f} H L n^{c}+\text { H.c. }
$$

where $L(H)$ represents the lepton (Higgs) doublet. In this equation the dimensionless parameter $\lambda$ is related to the neutrino mass as $\lambda=\sqrt{2} m_{\nu} / v_{\mathrm{EW}}$, where $v_{\mathrm{EW}}=246 \mathrm{GeV}$ is the electroweak vacuum expectation value. In the case when neutrinos are Majorana, we have instead

$$
i \frac{J}{f} \frac{(H L)^{2}}{\Lambda}+\text { H.c. }
$$

Here $\Lambda$ is an ultraviolet scale that is related to the neutrino mass as $\Lambda^{-1}=m_{\nu} / v_{\mathrm{EW}}^{2}$. Equations (7) and (8) are the leading order interactions of the Majoron with neutrinos in an expansion in $1 / f$. At low energies these reduce to Eqs. (1) and (2) respectively.

Assuming $m_{J} \gg m_{\nu}$, we obtain the total decay width of the Majoron to neutrinos as

$$
\Gamma_{J}^{D}=\frac{m_{\nu}^{2}}{f^{2}} \frac{m_{J}}{8 \pi} \quad \text { or } \quad \Gamma_{J}^{M}=\frac{m_{\nu}^{2}}{f^{2}} \frac{m_{J}}{16 \pi},
$$

where $\Gamma_{J}^{D}$ and $\Gamma_{J}^{M}$ correspond to the Dirac and Majorana cases respectively. We focus on Majoron masses $m_{J}$ of the order of an $\mathrm{eV}$ and Majoron lifetimes $\tau_{J}$ of the order of the age of the Universe. This translates to a scale $f$ of order $10^{6} \mathrm{GeV}$.

The Majoron model is constrained by astrophysical, cosmological and laboratory data. These bounds can be translated into limits on the mass of the Majoron $m_{J}$ and the decay constant $f$. For light Majorons, with masses less than a $\mathrm{MeV}$, the bounds from cosmology are the most severe. We therefore begin by considering the cosmological limits. Precision measurements of the CMB place limits on the total energy density in radiation during the epoch of acoustic oscillations. Additional energy density in radiation beyond the SM prediction is generally parametrized in terms of the effective number of neutrinos, $\Delta N_{\text {eff }}$. The CMB constraint, $\Delta N_{\text {eff }}<0.3$ [29], translates into the requirement that the Majoron not have a thermal abundance 
at temperatures of the order of a MeV, when the weak interactions decouple. For low values of $f$, Majoronneutrino scattering will bring the Majoron into thermal equilibrium with the neutrinos. To satisfy the bound, the decay constant $f$ must lie above $100 \mathrm{MeV}$ for $m_{J} \lesssim 1 \mathrm{MeV}$ in the case of Majorana neutrinos [34]. ${ }^{4}$ The limits in the Dirac case, though somewhat model dependent, are comparable.

For Majorons with masses around $\mathrm{eV}$, an even more severe constraint arises from the requirement that the Majoron not be in thermal equilibrium with the neutrinos during the $\mathrm{CMB}$ epoch. Inverse decays into Majorons prevent the neutrinos from free streaming, impacting the heights and locations of the CMB peaks. Current bounds indicate that neutrinos must be free streaming at temperatures $T$ of the order of an $\mathrm{eV}[37,38]$. This translates into a constraint on the Majoron decay constant, $f \gtrsim 100 \mathrm{GeV}$ [34], in the case of Majorana neutrinos. Again, the limits in the Dirac case, though somewhat model dependent, are similar.

So far we have focused on the cosmological limits arising from the dominant decay channel of Majorons, the decay to two neutrinos. Other subdominant channels, such as decays to charged leptons and photons, can also be used to set limits on $f$ because the bounds on fluxes of visible particles are usually stronger. Since our focus is on Majoron masses of order an $\mathrm{eV}$, the only relevant channel is $J \rightarrow \gamma \gamma$. However, for light Majorons the branching ratio to photons is extremely small, and the resulting bounds are very weak $[18,39,40]$.

The strongest astrophysical bounds arise from the effects of Majorons on supernovae. Neutrinos in a supernova receive corrections to their masses from matter effects, which are largest for electron neutrinos. Then, if the Majoron is light, electron neutrinos can decay into lighter flavors of neutrinos and Majorons. This can affect a supernova in two distinct ways. Prior to the bounce, these decays can deleptonize the core, preventing the explosion from taking place. After the bounce, the Majorons produced in these decays can free stream out, leading to overly rapid energy loss. While the resulting constraints depend on the details of supernova dynamics, they are at the level of $f \gtrsim 100 \mathrm{keV}$ [41-45] and therefore weaker than the cosmological bounds.

It is also possible to place bounds on neutrino-Majoron couplings from laboratory experiments, such as neutrinoless double beta decay [46,47], meson decay [41,48], charged lepton decay [49] and tritium decay [50]. These constraints arise from the modification of the energy spectrum of visible final states due to Majoron emissions. In all these cases, the limits are weaker than astrophysical and cosmological bounds for light Majorons. Clearly,

\footnotetext{
${ }^{4}$ It was recently shown that the constraint on $f$ can be weaker for $m_{J} \sim 1 \mathrm{MeV}[35,36]$.
}

our benchmark values of $f \sim 10^{6} \mathrm{GeV}$ and $m_{J} \sim 1 \mathrm{eV}$ are safe from all current bounds.

\section{THE NEUTRINO FLUX FROM MAJORON DECAYS}

In this section we determine the flux and energy distribution of the neutrinos arising from Majoron decays, as a function of the initial abundance of the Majoron, its mass and lifetime. In the case of cold Majorons with lifetimes comparable to the current age of the Universe we expect a sizable galactic contribution to the flux, in addition to the extragalactic contribution. The galactic flux is monochromatic, with frequency equal to exactly half the Majoron mass. The diffuse extragalactic component of the flux, however, undergoes redshift as the Universe expands, resulting in an energy distribution that, although not monochromatic, is highly distinctive.

\section{A. Extragalactic neutrinos}

We first determine the number density of diffuse neutrinos arising from Majorons decaying outside the Milky Way. Assuming SM neutrinos are Majorana, the comoving number density of neutrinos and antineutrinos arising from Majoron decay in a time interval $d t$ is given by

$$
d\left(n_{\nu} a^{3}\right)=d\left(n_{\bar{\nu}} a^{3}\right)=-\frac{n_{J} a^{3}}{\tau_{J}} d t,
$$

where $a$ represents the scale factor and $n_{J} a^{3}$ the comoving number density of Majorons. Now $n_{J}$ evolves as

$$
n_{J}=n_{J 0} e^{-t / \tau_{J}},
$$

where $n_{J 0}$ is the initial number density of Majorons. If Majorons contribute a fraction $\mathcal{F}$ of the energy density in dark matter at early times, we have

$$
n_{J 0} a^{3}=\mathcal{F} \Omega_{\mathrm{dm}} \frac{\rho_{c}}{m_{J}} .
$$

Then, Eq. (10) becomes

$$
d\left(n_{\nu} a^{3}\right)=-\frac{\mathcal{F} \Omega_{\mathrm{dm}}}{\tau_{J}} \frac{\rho_{c}}{m_{J}} e^{-t / \tau_{J}} d t
$$

At times when the Universe is dominated by dark matter and dark energy, the Hubble expansion at redshift $z$ can be approximated as

$$
H(z)=H_{0} \sqrt{\Omega_{\Lambda}+(1+z)^{3} \Omega_{m}} .
$$

Here $\Omega_{\Lambda}$ and $\Omega_{m}$ denote the fractional contributions of dark energy and matter to the total energy density of the Universe, and we are working in the limit $\Omega_{\Lambda}+\Omega_{m}=1$. We can use 


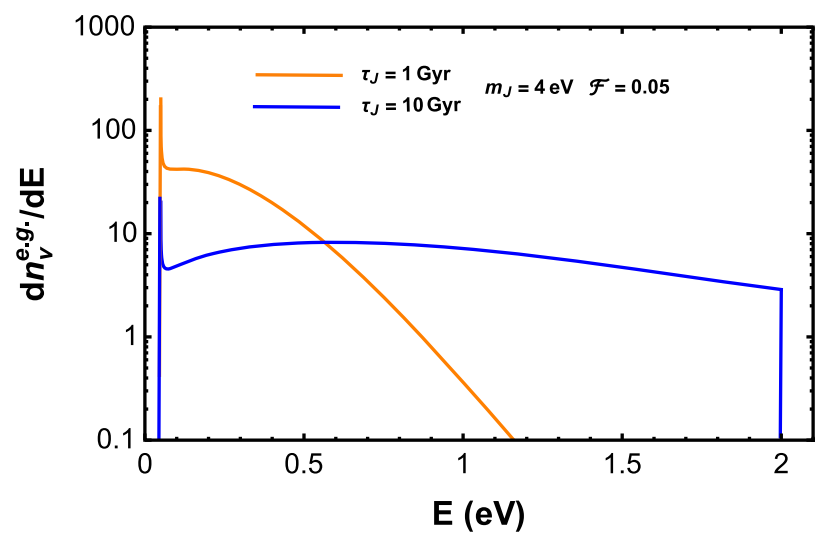

FIG. 1. The spectrum of the extragalactic neutrinos from Majoron decay for $m_{J}=4 \mathrm{eV}$ and $\mathcal{F}=0.05$ with two different lifetimes $\left(\tau_{J}=1\right.$ and $\left.10 \mathrm{Gyr}\right)$. The peak at $E=m_{\nu}=0.05 \mathrm{eV}$ corresponds to the fact that neutrinos from early decays of Majorons are all nonrelativistic with energies close to the neutrino mass. The spectrum also has a sharp edge at $E=m_{J} / 2$.

Eq. (14) to obtain an expression for the age of the Universe at redshift $z$ :

$$
t(z)=\frac{2}{3} \frac{1}{H_{0} \sqrt{\Omega_{\Lambda}}} \ln (\sqrt{r(z)}+\sqrt{1+r(z)}) .
$$

Here $r(z)$ represents the relative contributions of dark energy and matter to the total energy density of the Universe at redshift $z$,

$$
r(z)=\frac{\Omega_{\Lambda}}{\Omega_{m}} \frac{1}{(1+z)^{3}} .
$$

For neutrinos with energy $E$ today, we can infer that they were generated from Majorons that decayed at redshift $z_{E}$, where $z_{E}$ is given by

$1+z_{E}=\frac{p_{\max }}{\sqrt{E^{2}-m_{\nu}^{2}}} \quad$ with $\quad p_{\max } \equiv \sqrt{\frac{m_{J}^{2}}{4}-m_{\nu}^{2}}$.

Using Eqs. (15) and (17) to eliminate $t$ in favor of $E$ in Eq. (13), we can obtain an expression for the energy distribution of the extragalactic neutrinos from Majoron decay (see e.g., [20]),

$$
\begin{aligned}
\frac{d n_{\nu}^{\text {e.g. }}}{d E}= & \mathcal{F} \Omega_{\mathrm{dm}} \frac{\rho_{c}}{m_{J}} \frac{1}{\tau_{J} H_{0} \sqrt{\Omega_{\Lambda}+\Omega_{m}\left(1+z_{E}\right)^{3}}} e^{-\frac{t(z E)}{\tau_{J}}} \\
& \times \frac{E}{E^{2}-m_{\nu}^{2}}\left(E \in\left[m_{\nu}, \frac{m_{J}}{2}\right]\right) .
\end{aligned}
$$

The spectrum of neutrinos from extragalactic Majoron decay is shown in Fig. 1 for $m_{\nu}=0.05 \mathrm{eV}$ and two different values of the lifetime. For a given Majoron lifetime, the spectrum has sharp edges at $E=m_{J} / 2$ and
$E=m_{\nu}$, with a continuous distribution connecting these two points. The peak at $E=m_{\nu}$ corresponds to the fact that neutrinos from early decays of Majorons are all nonrelativistic with energies close to the neutrino mass.

\section{B. Galactic neutrinos}

If the Majoron lifetime is comparable to the age of the Universe, Majorons will clump inside the Milky Way galaxy. If the Majorons are sufficiently cold, their distribution in the galaxy will follow the cold dark matter profile. Since the time to traverse the galaxy is small compared to the age of the Universe, any neutrinos we observe from Majoron decay in the galaxy must have arisen from decays in the recent past. The spectrum of the galactic neutrinos produced from Majoron decays is therefore monochromatic, with energy $m_{J} / 2$. The flux of these neutrinos in the neighborhood of the Earth is given by the line of sight integral,

$$
n_{\nu}^{\mathrm{gal}} c=\mathcal{F} \frac{r_{\odot}}{\tau_{J}} \frac{\rho_{\odot}}{m_{J}} \bar{J} e^{-t_{0} / \tau_{J}},
$$

where $r_{\odot}=8.33 \mathrm{kpc}$ is the distance from the Earth to the Galactic Center and $\rho_{\odot}=0.3 \mathrm{GeV} / \mathrm{cm}^{3}$ is the dark matter density at the position of the Earth. Here the dimensionless factor $\bar{J}$ corresponds to the $J$ factor for the Navarro-FrenkWhite (NFW) profile [51] averaged over the Milky Way galaxy,

$$
\bar{J}=\frac{1}{4 \pi} \int_{-\pi / 2}^{\pi / 2} d b \cos b \int_{0}^{2 \pi} d \ell J^{\mathrm{NFW}}(b, \ell) \approx 2.19 .
$$

Here we have used galactic coordinates $(d, b, \ell)$ with distance from the Earth $d$, galactic latitude $b$ and longitude $\ell$. In this coordinate system, we can express any vector in the $(x, y, z)$ basis as

$x=d \cos b \cos \ell, \quad y=d \cos b \sin \ell, \quad z=d \sin b$.

The Earth lies at the center of this coordinate system while the Galactic Center is at $x=r_{\odot}$ and $y=z=0$. This means that the galactic plane is mapped to $z=0$, the $x y$-plane. $J^{\mathrm{NFW}}(b, \ell)$ is the $J$ factor for the NFW profile expressed as a function of $b$ and $\ell$ [52].

\section{DIRECT DETECTION IN NEUTRINO CAPTURE EXPERIMENTS}

The neutrinos from Majoron decay can be directly detected in experiments based on neutrino capture on nuclei. In this section we determine the size of the signal in experiments based on neutrino capture on tritium, with a focus on the PTOLEMY experiment. 
The PTOLEMY experiment has been designed to detect the $\mathrm{C} \nu \mathrm{B}$. The target consists of 100 grams of tritium coated on a graphene substrate. Tritium undergoes beta decay to $\mathrm{He}^{3}$ with a lifetime of 12.32 years, liberating $18.6 \mathrm{keV}$ of energy. This decay can also be induced by incident electron neutrinos, in which case the liberated electrons will have an energy that exceeds the end point energy of the beta decay process, allowing them to be distinguished. In the case of a nonrelativistic neutrino with mass $m_{\nu}$, the energy of the liberated electrons will exceed the end point energy by $2 m_{\nu}$. Since PTOLEMY has an energy resolution of about $0.15 \mathrm{eV}$, and the $\mathrm{C} \nu \mathrm{B}$ neutrinos are nonrelativistic, it is sensitive to $\mathrm{C} \nu \mathrm{B}$ neutrinos with masses above $0.1 \mathrm{eV}$. In contrast, the neutrinos from the decays of Majorons with masses in the $\mathrm{eV}$ range and lifetimes of the order of the age of the Universe are expected to be relativistic. We therefore expect that PTOLEMY will be able to detect these neutrinos, even if their masses lie well below $0.1 \mathrm{eV}$, provided their energies today are above $0.2 \mathrm{eV}$.

For low energy neutrinos the cross section $\sigma$ scales as the inverse of the relative velocity $v$, so that the product $\sigma v$ is a constant. The unpolarized capture rate of electron neutrinos on tritium is given by [27]

$$
\begin{aligned}
n_{\nu} \sigma v_{\nu} & =\left[\left(1-v_{\nu}\right) n_{\nu_{h_{R}}}+\left(1+v_{\nu}\right) n_{\nu_{h_{L}}}\right] \bar{\sigma}, \\
\bar{\sigma} & =3.83 \times 10^{-45} \mathrm{~cm}^{2},
\end{aligned}
$$

where $v_{\nu}$, the magnitude of the neutrino velocity, is close to 1 for relativistic neutrinos. The number density of left-helical (right-helical) neutrinos is denoted as $n_{\nu_{h_{L}\left(h_{R}\right)}}$. If neutrinos are Majorana (Dirac), the number density of neutrinos from Majoron decay is $n_{\nu_{h_{L}}}=n_{\nu_{h_{R}}}=n_{\nu}^{J}\left(n_{\nu_{h_{L}}}=\right.$ $\frac{1}{2} n_{\nu}^{J}, n_{\nu_{h_{R}}}=\frac{1}{2} n_{\nu}^{J}$ ). Equation (22) is valid for incident neutrinos with energies below a few $\mathrm{keV}$. It is clear from this formula that, for a given Majoron mass and lifetime, the signal rate from Majorana neutrinos (denoted as $R_{\mathrm{M}}$ ) is larger by a factor of 2 than the rate from Dirac neutrinos (denoted as $R_{\mathrm{D}}$ ), $R_{\mathrm{M}}=2 R_{D}$. Neutrinos from galactic and extragalactic Majoron decays exhibit characteristic energy distributions, and the signal event rate at PTOLEMY will maintain the relation $R_{\mathrm{M}}=2 R_{D}$ throughout the entire spectrum.

Only neutrinos in the electron flavor can be captured on tritium. Since the neutrinos produced in Majoron decay are in mass eigenstates, the event rate in PTOLEMY depends on the neutrino masses and mixing angles. The probability that a neutrino from Majoron decay is in the eigenstate with mass $m_{i}$ is given by

$$
P_{i}=\frac{m_{i}^{2}}{m_{1}^{2}+m_{2}^{2}+m_{3}^{2}} .
$$

The capture cross section for a neutrino in the $i$ th mass eigenstate is smaller by a factor of $\left|U_{e i}\right|^{2}$ than that for an electron neutrino. It follows that the overall number of

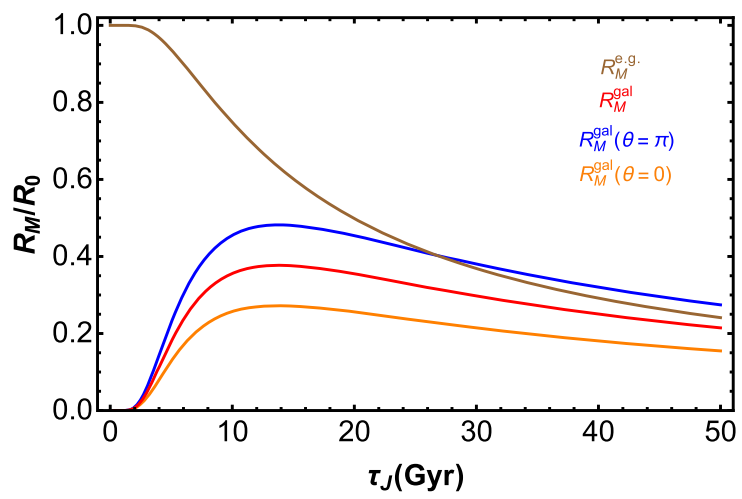

FIG. 2. The signal rate normalized to $R_{0}$ [Eq. (25)] as a function of Majoron lifetime $\left(\tau_{J}\right)$ from the extragalactic neutrino source $\left(R_{M}^{\text {e.g. }}\right)$, the averaged galactic neutrinos $\left(R_{M}^{\mathrm{gal}}\right)$, galactic neutrinos with tritium spin polarized towards $\left[R_{M}^{\mathrm{gal}}(\theta=0)\right]$ and away $\left[R_{M}^{\mathrm{gal}}(\theta=\pi)\right]$ from the Galactic Center.

events from Majoron decay is suppressed by an overall factor of $\sum_{i}\left|U_{e i}^{2}\right| P_{i}$ relative to that from an equivalent flux of purely electron neutrinos. The current best fits to (three flavor) neutrino data yield $\left|U_{e 1}\right|^{2}=0.67,\left|U_{e 2}\right|^{2}=0.30$ and $\left|U_{e 3}\right|^{2}=0.022$. The solar and atmospheric mass splittings are given by $(0.0086 \mathrm{eV})^{2}$ and $(0.050 \mathrm{eV})^{2}$ respectively [53]. Then, if the lightest neutrino is massless, the suppression factor is about 0.03 in the case of a normal hierarchy and about $1 / 2$ in the case of an inverted hierarchy. If neutrinos are quasidegenerate, the suppression factor is about $1 / 3$.

The number of extragalactic (galactic) neutrino events per year expected in PTOLEMY, in the case of Majorana neutrinos, is given by (see Fig. 2)

$R_{M}^{\text {e.g. }}=R_{0}\left(1-e^{-t_{0} / \tau_{J}}\right), \quad R_{M}^{\mathrm{gal}}=R_{0} \frac{r_{\odot} \rho_{\odot}}{\tau_{J} \Omega_{\mathrm{dm}} \rho_{c}} \bar{J} e^{-t_{0} / \tau_{J}}$

where $R_{0}$ is given by

$$
\begin{aligned}
R_{0}= & N_{H} 2 n_{\nu 0}^{J} \sum_{i}\left|U_{e i}^{2}\right| P_{i} \bar{\sigma}=\frac{4.6}{\text { year }} \frac{\mathcal{F}}{5 \%} \frac{\mathrm{eV}}{m_{J}} \frac{\sum_{i}\left|U_{e i}^{2}\right| P_{i}}{1 / 2} \\
& \times \frac{M_{T}}{100 \mathrm{~g}} \frac{\bar{\sigma}}{3.83 \times 10^{-45} \mathrm{~cm}^{2}} .
\end{aligned}
$$

Here $N_{\mathrm{H}}$ is the number of tritium nuclei in the detector and $M_{T}$ is the mass of the tritium target. We see that in the case of quasidegenerate and inverted spectra, we can expect to see as many as a few extragalactic events per year, provided the neutrino energies are above the detector threshold. This falls to one event every few years in the case of a normal hierarchy. Note that this signal rate is comparable to

${ }^{5}$ We use the latest 2018 data for the normal hierarchy from NuFIT 4.0 (2018), www.nu-fit.org. 
the expected event rate for the standard $\mathrm{C} \nu \mathrm{B}$, which corresponds to 4 (8) events at PTOLEMY if neutrinos are Dirac (Majorana). ${ }^{6}$

\section{A. Extragalactic neutrino spectrum}

Since extragalactic neutrinos are approximately isotropic, we can use the unpolarized cross section in Eq. (22). By combining Eqs. (18) and (22), we obtain the event rate for Majorana neutrinos,

$$
\begin{aligned}
\frac{1}{R_{0}} \frac{d R_{M}^{\text {e.g. }}}{d E}= & \frac{1}{\tau_{J} H_{0} \sqrt{\Omega_{\Lambda}+\Omega_{m}\left(1+z_{E}\right)^{3}}} e^{-\frac{t\left(z_{E}\right)}{\tau_{J}}} \frac{E}{E^{2}-m_{\nu}^{2}} \\
& \times\left(E \in\left[m_{\nu}, \frac{m_{J}}{2}\right]\right)
\end{aligned}
$$

where $z_{E}$ is defined in Eq. (17).

By integrating Eq. (26), $R_{M}^{\text {e.g. }}=\int d E \frac{d d_{M}^{\text {e.g. }}}{d E}=R_{0}\left(1-e^{-t_{0} / \tau_{J}}\right)$, we reproduce the result for the total event rate. If the lifetime is smaller than the age of the Universe $\left(\tau_{J}<t_{0}\right)$, most of the Majorons will have decayed. Then the event rate is expected to be close to $R_{0}, R_{M}^{\text {e.g. }} \approx R_{0}$, provided the neutrinos retain enough energy to pass the detector threshold. If, however, the lifetime is longer than the age of the Universe, the fraction of Majorons that have decayed before today is smaller. Then the ratio $R_{M}^{\text {e.g. }} / R_{0}$ gets smaller as $\tau_{J}$ increases, as can be seen in Fig. 2.

To obtain a realistic spectrum with finite experimental resolution, we apply a Gaussian smearing with the full width at half maximum (FWHM) $\Delta$ defined as

$$
\Delta=\sqrt{8 \ln 2} \sigma,
$$

where $\sigma$ is the standard deviation of the Gaussian. The expected energy resolution of PTOLEMY, $\Delta=0.15 \mathrm{eV}$, corresponds to a standard deviation $\sigma=0.064 \mathrm{eV}$. With this Gaussian smearing, the extragalactic neutrino spectrum is shown in Fig. 3. In this figure, we assume that neutrino masses exhibit an inverted hierarchy with the lightest eigenstate being massless. This means the two massive states both have masses around $0.05 \mathrm{eV}$. Moreover, the electron flavored state $\nu_{e}$ is mostly a linear combination of the two massive states. Therefore, we show the signal spectrum for a single value $m_{\nu}=0.05 \mathrm{eV}$. Note that the neutrino energy is related to the kinetic energy of the emitted electron $K_{e}$ as $E=K_{e}-K_{\text {end }}^{0}$, where $K_{\text {end }}^{0}$ is the beta decay end point energy if $m_{\nu}=0$.

\section{B. Polarized tritium and the galactic neutrino spectrum}

The capture cross section of neutrinos on tritium depends on the relative angle between the incoming neutrino

\footnotetext{
${ }^{6}$ Gravitational clustering of nonrelativistic neutrinos is expected to modify the $\mathrm{C} \nu \mathrm{B}$ event rate, but only by order 1 factors $[54,55]$.
}

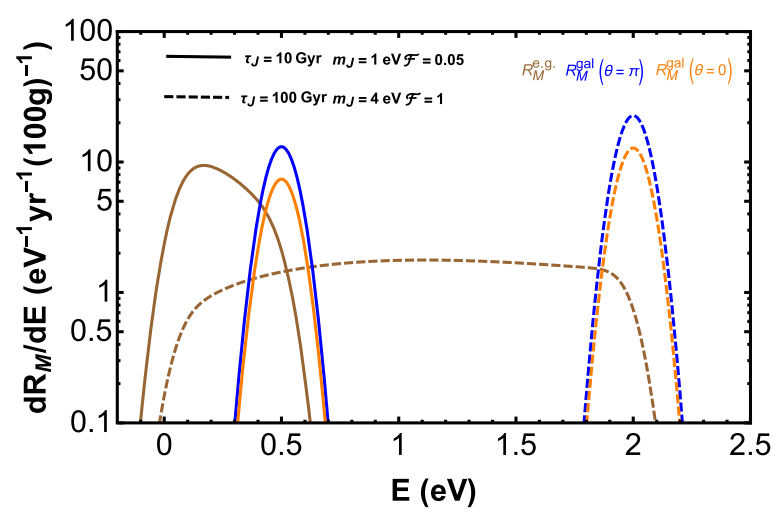

FIG. 3. This plot shows $d R_{M} / d E$ as a function of neutrino energy $E$ for two benchmark points on a $100 \mathrm{~g}$ tritium target for $m_{\nu}=0.05 \mathrm{eV}$, assuming neutrino masses exhibit an inverted hierarchy. Solid lines represent the benchmark $\left(m_{J}=1 \mathrm{eV}, \tau_{J}=\right.$ 10 Gyr and $\mathcal{F}=0.05$ ) while the dashed lines show the benchmark $\left(m_{J}=4 \mathrm{eV}, \tau_{J}=100 \mathrm{Gyr}\right.$ and $\left.\mathcal{F}=1\right)$. We show the energy spectra from extragalactic neutrino decays (brown curves), galactic neutrino decays with tritium spin polarized towards $(\theta=0$, orange) and away $(\theta=\pi$, blue) from the Galactic Center. Here we apply $\sigma=0.064 \mathrm{eV}$ (from the experimental resolution $\Delta=\sqrt{8 \ln 2} \sigma=0.15 \mathrm{eV}$ ) Gaussian smearing. These results are for Majorana neutrinos. The rates for Dirac neutrinos are half of those in the Majorana case.

velocity and the spin of the tritium nucleus. As discussed in [33], this means that if the tritium used in PTOLEMY is polarized, we can obtain information about the direction of the neutrino signal. This in turn can be used to obtain information about the distribution of the Majoron component of dark matter in the galaxy. Since the galactic neutrinos will have energies of the order of an $\mathrm{eV}$, they can be treated as ultrarelativistic. Averaging over the angles of the outgoing electrons, one obtains the polarized capture rate for relativistic electron neutrinos as [33]

$$
n_{\nu} \sigma(\hat{\mathbf{s}}, \hat{\mathbf{v}}) v=2 n_{\nu_{h_{L}}} \bar{\sigma}(1+B \hat{\mathbf{S}} \cdot \hat{\mathbf{v}}) .
$$

Here the parameter $B \approx 0.99$ incorporates the relevant couplings and form factors. The unit vector in the direction of the tritium spin is denoted as $\hat{\mathbf{s}}$, while the direction of the incoming neutrino is $\hat{\mathbf{v}}$. As mentioned earlier, $n_{\nu_{h_{L}}}=n_{\nu}^{J}$ if neutrinos are Majorana, while $n_{\nu_{h_{L}}}=\frac{1}{2} n_{\nu}^{J}$ if neutrinos are Dirac. Since the distribution of extragalactic sources (discussed in Sec. III A) is expected to be isotropic, the result for that case simply reduces to Eq. (22) after averaging over the tritium spin in the relativistic limit. However, because the galactic distribution of cold Majorons follows a NFW profile, interesting directional information is encoded in a term proportional to $\hat{\mathbf{s}} \cdot \hat{\mathbf{v}}$ in Eq. (28).

We wish to determine the dependence of the signal on the orientation of the spin of the tritium nuclei relative to the Galactic Center. We begin by writing $\hat{\mathbf{s}} \cdot \hat{\mathbf{v}}$ in terms of galactic coordinates, 


$$
\hat{\mathbf{s}} \cdot \hat{\mathbf{v}}=\cos b_{\hat{\mathbf{s}}} \cos b_{\hat{\mathbf{v}}} \cos \left(\ell_{\hat{\mathbf{v}}}-\ell_{\hat{\mathbf{s}}}\right)+\sin b_{\hat{\mathbf{s}}} \sin b_{\hat{\mathbf{v}}},
$$

where $\hat{\mathbf{s}}(\hat{\mathbf{v}})=\cos b_{\hat{\mathbf{s}}(\hat{\mathbf{v}})} \cos \ell_{\hat{\mathbf{s}}(\hat{\mathbf{v}})} \hat{\mathbf{e}}_{x}+\cos b_{\hat{\mathbf{s}}(\hat{\mathbf{v}})} \sin \ell_{\hat{\mathbf{s}}(\hat{\mathbf{v}})} \hat{\mathbf{e}}_{y}+$ $\sin b_{\hat{\mathbf{s}}(\hat{\mathbf{v}})} \hat{\mathbf{e}}_{z}$ with $\hat{\mathbf{e}}_{x, y, z}$ being the unit vectors along the $x$, $y$ and $z$ directions. We also define $\theta$ as the angle between the tritium spin and the unit vector pointing towards the Galactic Center $\left(\hat{\mathbf{e}}_{x}\right)$,

$$
\cos \theta \equiv \hat{\mathbf{s}} \cdot \hat{\mathbf{e}}_{x}=\cos b_{\hat{\mathbf{s}}} \cos \ell_{\hat{\mathbf{s}}}
$$

Since the form of the NFW distribution is invariant under rotations along the line connecting the Earth and the Galactic Center, the final result should also be unchanged under such a rotation. This allows us to choose coordinates such that $\ell_{\hat{\mathbf{s}}}=0$ or $\pi$. Combining Eqs. (29) and (30), we obtain

$$
\begin{aligned}
\hat{\mathbf{s}} \cdot \hat{\mathbf{v}} & =\left\{\begin{array}{l}
\cos b_{\hat{\mathbf{s}}} \cos b_{\hat{\mathbf{v}}} \cos \ell_{\hat{\mathbf{v}}}+\sin b_{\hat{\mathbf{s}}} \sin b_{\hat{\mathbf{v}}}\left(\ell_{\hat{\mathbf{s}}}=0\right) \\
-\cos b_{\hat{\mathbf{s}}} \cos b_{\hat{\mathbf{v}}} \cos \ell_{\hat{\mathbf{v}}}+\sin b_{\hat{\mathbf{s}}} \sin b_{\hat{\mathbf{v}}}\left(\ell_{\hat{\mathbf{s}}}=\pi\right)
\end{array}\right. \\
& =\cos \theta \cos b_{\hat{\mathbf{v}}} \cos \ell_{\hat{\mathbf{v}}}+\sin \theta \sin b_{\hat{\mathbf{v}}} .
\end{aligned}
$$

In this case of Majorana neutrinos, the rate when the spin is oriented at an angle $\theta$ is given by

$$
\begin{aligned}
\frac{R_{M}^{\mathrm{gal}}(\theta)}{R_{0}}= & \frac{r_{\odot} \rho_{\odot}}{\tau_{J} \Omega_{\mathrm{dm}} \rho_{c}} e^{-t_{0} / \tau_{J}}\left[\bar{J}+\frac{B}{4 \pi} \int_{-\pi / 2}^{\pi / 2} d b \int_{0}^{2 \pi} d \ell \cos b\right. \\
& \left.\times(-\cos \theta \cos b \cos \ell-\sin \theta \sin b) J^{\mathrm{NFW}}(b, \ell)\right] \\
\approx & \frac{r_{\odot} \rho_{\odot}}{\tau_{J} \Omega_{\mathrm{dm}} \rho_{c}} e^{-t_{0} / \tau_{J}}(2.19-0.60 \cos \theta) .
\end{aligned}
$$

It is clear from Eq. (32) that the galactic signal varies by almost a factor of 2 depending on whether the spin of the polarized tritium points towards or away from the Galactic Center,

$$
\frac{R_{M}^{\mathrm{gal}}(\theta=\pi)}{R_{M}^{\mathrm{gal}}(\theta=0)} \approx 1.8 .
$$

This is a characteristic feature of the galactic neutrino signal. PTOLEMY with polarized tritium can test this prediction by measuring the modulation associated with the orientation of the spin. ${ }^{7}$

In principle, all galactic neutrinos have the same energy $E=m_{J} / 2$. However, due to experimental resolution, the actual spectrum of galactic neutrinos will be smeared out. Including the Gaussian smearing with $\sigma=0.064 \mathrm{eV}$ mentioned before, the galactic neutrino spectrum is given by

\footnotetext{
${ }^{7}$ Polarized tritium can also be used to measure the annual modulation of PTOLEMY signals, both in the context of the standard $\mathrm{C} \nu \mathrm{B}$ [56] and of new physics [57].
}

$$
\begin{aligned}
\frac{1}{R_{0}} \frac{d R_{M}^{\mathrm{gal}}(\theta)}{d E}= & \frac{1}{\sqrt{2 \pi} \sigma} \frac{n_{0}^{\mathrm{gal}}}{n_{\nu 0}^{J}}(2.19-0.60 \cos \theta) \\
& \times \exp \left[-\frac{\left(E-\frac{m_{J}}{2}\right)^{2}}{2 \sigma^{2}}\right]
\end{aligned}
$$

where

$$
\frac{n_{0}^{\mathrm{gal}}}{n_{\nu 0}^{J}}=\frac{r_{\odot} \rho_{\odot}}{\tau_{J} \Omega_{\mathrm{dm}} \rho_{c}} e^{-t_{0} / \tau_{J}} .
$$

The galactic neutrino spectrum is shown in Fig. 3. From the above expression, we see that the total signal rate from galactic neutrinos is proportional to $e^{-t_{0} / \tau_{J}} / \tau_{J}$. Therefore, $R_{M}^{\mathrm{gal}}$ is peaked at $\tau_{J}=t_{0}$, as can be seen in Fig. 2. If $\tau_{J} \ll t_{0}$ the event rate is small due to the galactic Majoron number density being suppressed as $e^{-t_{0} / \tau_{J}}$. On the other hand, if $\tau_{J} \gg t_{0}$, the signal is suppressed because of the small decay rate.

Comparing the signals from extragalactic and galactic Majoron decay, we see that both signals can be easily distinguished from each other, and from $\mathrm{C} \nu \mathrm{B}$ signals. $\mathrm{C} \nu \mathrm{B}$ signals accumulate close to $E=m_{\nu}$ while the energy distribution of neutrinos from extragalactic decays exhibits a distinctive spectrum, as shown in Fig. 3. Galactic signals can be distinguished by their monochromatic nature, and, if the tritium target is polarized, by employing directional information.

The directional dependence of the signal also encodes information about the dark matter distribution in the Milky Way galaxy. Once $R_{M}^{\mathrm{gal}}(\theta)$ is measured, the ratio of the $\theta$ independent term to the coefficient of the $\cos \theta$ term in Eq. (32) captures information about the distribution of dark matter. Measuring this ratio would help discriminate between models of the dark matter profile in the Milky Way.

\section{Benchmark points}

For concreteness we consider two benchmark points. We first choose $m_{J}=1 \mathrm{eV}$ and a lifetime that is lightly shorter than the age of the Universe, $\tau_{J}=10 \mathrm{Gyr}$. In this case the current bounds from decaying dark matter indicate that Majorons can only contribute about $5 \%$ of the total dark matter abundance [21]. Therefore, the first benchmark point we choose is BP1: $m_{J}=1 \mathrm{eV}, \tau_{J}=10 \mathrm{Gyr}$ and $\mathcal{F}=0.05$. From the analysis in [21], if the lifetime of the Majoron is sufficiently longer than the age of the Universe, say $\tau_{J}=100 \mathrm{Gyr}$, it can constitute all of the dark matter abundance, so that $\mathcal{F}=1$. Therefore we consider a second benchmark BP2: $m_{J}=4 \mathrm{eV}, \tau_{J}=100 \mathrm{Gyr}$ and $\mathcal{F}=1$. For these two benchmark points with quasidegenerate neutrino masses, we obtain the total signal rates from extragalactic decays, Eq. (24), and galactic decays with a polarized tritium target, Eq. (32), as 


$$
\begin{aligned}
\mathrm{BP} 1: R_{M}^{\text {e.g. }} & =3.4 /(100 \mathrm{~g} \cdot \mathrm{yr}), \\
R_{M}^{\mathrm{gal}}(\theta=\pi) & =2.1 /(100 \mathrm{~g} \cdot \mathrm{yr}) \\
\mathrm{BP} 2: R_{M}^{\text {e.g. }} & =3.0 /(100 \mathrm{~g} \cdot \mathrm{yr}), \\
R_{M}^{\mathrm{gal}}(\theta=\pi) & =3.6 /(100 \mathrm{~g} \cdot \mathrm{yr}) .
\end{aligned}
$$

The dominant background at PTOLEMY arises from regular beta decay events in which the electron carries away almost all the liberated energy. We expect that this background falls off very quickly for $E>0.2 \mathrm{eV}$ in Fig. 3. The basis of this expectation is that PTOLEMY experiments have sensitivity to $\mathrm{C} \nu \mathrm{B}$ neutrinos with $m_{\nu} \gtrsim 0.1 \mathrm{eV}$, which means that the experiment must be able to discriminate events with electron energy $0.2 \mathrm{eV}$ or more above the end point of the beta decay spectrum. Following this assumption, we obtain the sum of extragalactic and galactic event rates above $E \gtrsim 0.2 \mathrm{eV}$ to be $R_{M}^{\text {tot }}=4.0$ for BP1 and $R_{M}^{\text {tot }}=6.5$ for BP2 with 100 grams of tritium and 1 year exposure. Therefore, we expect to have a few signal events with a specific energy spectrum in the small background range with just 1 year of data. Clearly, observing such a striking signal would provide strong evidence of Majoron decays.

\section{CONCLUSION}

In this paper we have analyzed the prospects for discovery of a secondary cosmic neutrino background originating from Majoron decays at future detectors based on neutrino capture on tritium, such as PTOLEMY. We have studied the bounds on this scenario and determined the event rate and energy distribution of the signal as a function of the Majoron mass and lifetime. We find that for Majoron masses at the $\mathrm{eV}$ scale and lifetimes of the order of the age of the Universe, these detectors will be sensitive to this class of models with 100 gram-years of data. We have considered two distinct sources of this signal, galactic and extragalactic, and find that each exhibits a distinctive spectrum that can be distinguished using the data. Finally, we have explored the possibility of using polarized tritium to obtain directional information about the incoming neutrinos. This can be used to discriminate between neutrinos with galactic and extragalactic origins and to shed light on the distribution of dark matter in our galaxy.

\section{ACKNOWLEDGMENTS}

We would like to thank Oren Slone for useful discussions. Z. C., P. D. and M. G. are supported in part by the National Science Foundation under Grant No. PHY1620074. Z. C. would like to thank the Fermilab Theory Group for hospitality during the completion of this work. Z. C.'s stay at Fermilab was supported by the Fermilab Intensity Frontier Fellowship and by the Visiting Scholars Grant No. 17-S-02 from the Universities Research Association.

Note added.-Recently, we received Ref. [58], which discusses ideas similar to those considered here. Both our work and Ref. [58] consider the signals of extragalactic and galactic neutrinos from Majoron decays at neutrino capture experiments (e.g., PTOLEMY). However, Ref. [58] includes a discussion of the signals and CMB constraints in the case that the Majorons decay prior to recombination, which is not discussed in our paper. Our work points out that directional information about the galactic signal can be obtained if the target tritium is polarized, which is not discussed in Ref. [58].
[1] P. Minkowski, $\mu \rightarrow e \gamma$ at a rate of one out of $10^{9}$ muon decays, Phys. Lett. 67B, 421 (1977).

[2] T. Yanagida, Proceedings of the Workshop on Unified Theories, KEK Report 79-18 (1979), p. 95.

[3] S. L. Glashow, 1979 Cargese Summer Institute on Quarks and Leptons (Plenum, New York, 1980), p. 687.

[4] M. Gell-Mann, P. Ramond, and R. Slansky, Complex spinors and unified theories, Conf. Proc. C790927, 315 (1979).

[5] R. N. Mohapatra and G. Senjanovic, Neutrino masses and mixings in gauge models with spontaneous parity violation, Phys. Rev. D 23, 165 (1981).

[6] G. B. Gelmini and M. Roncadelli, Left-handed neutrino mass scale and spontaneously broken lepton number, Phys. Lett. 99B, 411 (1981).
[7] Y. Chikashige, R. N. Mohapatra, and R. D. Peccei, Are there real Goldstone bosons associated with broken lepton number, Phys. Lett. 98B, 265 (1981).

[8] H. M. Georgi, S. L. Glashow, and S. Nussinov, Unconventional model of neutrino masses, Nucl. Phys. B193, 297 (1981).

[9] I. Z. Rothstein, K. S. Babu, and D. Seckel, Planck scale symmetry breaking and majoron physics, Nucl. Phys. B403, 725 (1993).

[10] V. Berezinsky and J. W. F. Valle, The keV majoron as a dark matter particle, Phys. Lett. B 318, 360 (1993).

[11] M. Frigerio, T. Hambye, and E. Masso, Sub-GeV Dark Matter as Pseudo-Goldstone from the Seesaw Scale, Phys. Rev. X 1, 021026 (2011). 
[12] N. Rojas, R. A. Lineros, and F. Gonzalez-Canales, Majoron dark matter from a spontaneous inverse seesaw model, arXiv:1703.03416.

[13] T. Brune and H. Päs, Majoron dark matter and constraints on the Majoron-neutrino coupling, Phys. Rev. D 99, 096005 (2019).

[14] J. Preskill, M. B. Wise, and F. Wilczek, Cosmology of the invisible axion, Phys. Lett. 120B, 127 (1983).

[15] L. F. Abbott and P. Sikivie, A cosmological bound on the invisible axion, Phys. Lett. 120B, 133 (1983).

[16] M. Dine and W. Fischler, The not so harmless axion, Phys. Lett. 120B, 137 (1983).

[17] S. Palomares-Ruiz, Model-independent bound on the dark matter lifetime, Phys. Lett. B 665, 50 (2008).

[18] C. Garcia-Cely and J. Heeck, Neutrino lines from majoron dark matter, J. High Energy Phys. 05 (2017) 102.

[19] L. Covi, M. Grefe, A. Ibarra, and D. Tran, Neutrino signals from dark matter decay, J. Cosmol. Astropart. Phys. 04 (2010) 017.

[20] Y. Cui, M. Pospelov, and J. Pradler, Signatures of dark radiation in neutrino and dark matter detectors, Phys. Rev. D 97, 103004 (2018).

[21] V. Poulin, P. D. Serpico, and J. Lesgourgues, A fresh look at linear cosmological constraints on a decaying dark matter component, J. Cosmol. Astropart. Phys. 08 (2016) 036.

[22] A. Chudaykin, D. Gorbunov, and I. Tkachev, Dark matter component decaying after recombination: Lensing constraints with Planck data, Phys. Rev. D 94, 023528 (2016).

[23] A. Chudaykin, D. Gorbunov, and I. Tkachev, Dark matter component decaying after recombination: Sensitivity to baryon acoustic oscillation and redshift space distortion probes, Phys. Rev. D 97, 083508 (2018).

[24] S. Weinberg, Universal neutrino degeneracy, Phys. Rev. 128, 1457 (1962).

[25] S. Betts et al., Development of a relic neutrino detection experiment at PTOLEMY: Princeton tritium observatory for light, early-universe, massive-neutrino yield, in Proceedings of the 2013 Community Summer Study on the Future of U.S. Particle Physics: Snowmass on the Mississippi (CSS2013): Minneapolis, MN, USA, 2013 (2013).

[26] E. Baracchini et al. (PTOLEMY Collaboration), PTOLEMY: A proposal for thermal relic detection of massive neutrinos and directional detection of $\mathrm{MeV}$ dark matter, arXiv:1808 .01892 .

[27] A. J. Long, C. Lunardini, and E. Sabancilar, Detecting non-relativistic cosmic neutrinos by capture on tritium: Phenomenology and physics potential, J. Cosmol. Astropart. Phys. 08 (2014) 038.

[28] E. Roulet and F. Vissani, On the capture rates of big bang neutrinos by nuclei within the Dirac and Majorana hypotheses, J. Cosmol. Astropart. Phys. 10 (2018) 049.

[29] N. Aghanim et al. (Planck Collaboration), Planck 2018 results. VI. Cosmological parameters, arXiv:1807.06209.

[30] M.-C. Chen, M. Ratz, and A. Trautner, Nonthermal cosmic neutrino background, Phys. Rev. D 92, 123006 (2015).

[31] J. Zhang and S. Zhou, Relic right-handed Dirac neutrinos and implications for detection of cosmic neutrino background, Nucl. Phys. B903, 211 (2016).

[32] M. Arteaga, E. Bertuzzo, Y. F. Perez-Gonzalez, and R. Z. Funchal, Impact of beyond the Standard Model physics in the detection of the cosmic neutrino background, J. High Energy Phys. 09 (2017) 124.

[33] M. Lisanti, B. R. Safdi, and C. G. Tully, Measuring anisotropies in the cosmic neutrino background, Phys. Rev. D 90, 073006 (2014).

[34] Z. Chacko, L. J. Hall, T. Okui, and S. J. Oliver, CMB signals of neutrino mass generation, Phys. Rev. D 70, 085008 (2004).

[35] C. D. Kreisch, F.-Y. Cyr-Racine, and O. Doré, The neutrino puzzle: Anomalies, interactions, and cosmological tensions, arXiv:1902.00534.

[36] G. Barenboim, P. B. Denton, and I. M. Oldengott, Constraints on inflation with an extended neutrino sector, Phys. Rev. D 99, 083515 (2019).

[37] M. Archidiacono and S. Hannestad, Updated constraints on non-standard neutrino interactions from Planck, J. Cosmol. Astropart. Phys. 07 (2014) 046.

[38] F. Forastieri, M. Lattanzi, and P. Natoli, Constraints on secret neutrino interactions after Planck, J. Cosmol. Astropart. Phys. 07 (2015) 014.

[39] F. Bazzocchi, M. Lattanzi, S. Riemer-Sorensen, and J. W. F. Valle, X-ray photons from late-decaying majoron dark matter, J. Cosmol. Astropart. Phys. 08 (2008) 013.

[40] M. Lattanzi, S. Riemer-Sorensen, M. Tortola, and J. W. F. Valle, Updated CMB and $\mathrm{x}$ - and $\gamma$-ray constraints on Majoron dark matter, Phys. Rev. D 88, 063528 (2013).

[41] G. B. Gelmini, S. Nussinov, and M. Roncadelli, Bounds and prospects for the Majoron model of left-handed neutrino masses, Nucl. Phys. B209, 157 (1982).

[42] E. W. Kolb and M. S. Turner, Supernova SN 1987a and the secret interactions of neutrinos, Phys. Rev. D 36, 2895 (1987).

[43] K. Choi and A. Santamaria, Majorons and supernova cooling, Phys. Rev. D 42, 293 (1990).

[44] M. Kachelriess, R. Tomas, and J. W. F. Valle, Supernova bounds on Majoron emitting decays of light neutrinos, Phys. Rev. D 62, 023004 (2000).

[45] Y. Farzan, Bounds on the coupling of the Majoron to light neutrinos from supernova cooling, Phys. Rev. D 67, 073015 (2003).

[46] M. Doi, T. Kotani, and E. Takasugi, Double beta decay and Majorana neutrino, Prog. Theor. Phys. Suppl. 83, 1 (1985).

[47] M. Doi, T. Kotani, and E. Takasugi, The neutrinoless double beta decay with Majoron emission, Phys. Rev. D 37, 2575 (1988).

[48] V. D. Barger, W.-Y. Keung, and S. Pakvasa, Majoron emission by neutrinos, Phys. Rev. D 25, 907 (1982).

[49] A. P. Lessa and O. L. G. Peres, Revising limits on neutrinoMajoron couplings, Phys. Rev. D 75, 094001 (2007).

[50] G. Arcadi, J. Heeck, F. Heizmann, S. Mertens, F. S. Queiroz, W. Rodejohann, M. Slezák, and K. Valerius, Tritium beta decay with additional emission of new light bosons, J. High Energy Phys. 01 (2019) 206.

[51] J. F. Navarro, C. S. Frenk, and S. D. M. White, A universal density profile from hierarchical clustering, Astrophys. J. 490, 493 (1997).

[52] M. Cirelli, G. Corcella, A. Hektor, G. Hutsi, M. Kadastik, P. Panci, M. Raidal, F. Sala, and A. Strumia, PPPC 4 DM ID: A poor particle physicist cookbook for dark matter indirect 
detection, J. Cosmol. Astropart. Phys. 03 (2011) 051; Erratum, J. Cosmol. Astropart. Phys. 10 (2012) E01(E).

[53] I. Esteban, M. C. Gonzalez-Garcia, A. Hernandez-Cabezudo, M. Maltoni, and T. Schwetz, Global analysis of three-flavour neutrino oscillations: Synergies and tensions in the determination of $\theta_{2} 3, \delta_{C} P$, and the mass ordering, J. High Energy Phys. 01 (2019) 106.

[54] A. Ringwald and Y. Y. Y. Wong, Gravitational clustering of relic neutrinos and implications for their detection, J. Cosmol. Astropart. Phys. 12 (2004) 005.
[55] J. Zhang and X. Zhang, Gravitational clustering of cosmic relic neutrinos in the Milky Way, Nat. Commun. 9, 1833 (2018).

[56] B. R. Safdi, M. Lisanti, J. Spitz, and J. A. Formaggio, Annual modulation of cosmic relic neutrinos, Phys. Rev. D 90, 043001 (2014).

[57] G.-y. Huang and S. Zhou, Discriminating between thermal and nonthermal cosmic relic neutrinos through an annual modulation at PTOLEMY, Phys. Rev. D 94, 116009 (2016).

[58] D. McKeen, Cosmic neutrino background search experiments as decaying dark matter detectors, arXiv:1812.08178. 